\title{
Manejo del Niño Inmigrante: Calendario Vacunal
}

\author{
María Doyle Sáncheza , Josefa Plaza Almeida ${ }^{\text {, }}$ Fernando Mateos Rodríguez ${ }^{c}$, \\ María del Perpetuo Socorro Hoyos Vázqueza
}

a Médico Interno Residente de Pediatría. Complejo Hospitalario Universitario de Albacete.

b Pediatra de Atención Primaria. Centro de Salud Zona I de Albacete.

${ }^{c}$ Medicina Interna. Complejo Hospitalario Universitario de Albacete.

\section{Correspondencia:}

Josefa Plaza Almeida, Avda. Estación n 6, 9D, 02001-Albacete, España. Tfno: 967247565,

e-mail: plazaalmeida@hotmail.com

Recibido el 25 de noviembre de 2008

Aceptado para su publicación el 14 de diciembre de 2008

\section{RESUMEN}

En Castilla-La Mancha la población extranjera se ha multiplicado en el último quinquenio. La progresión de la incorporación de inmigrantes ha sido muy rápida en los últimos años, con incremento más acusado a partir del año 2001. Los inmigrantes proceden a menudo de comunidades socioeconómicas deprimidas, siendo la población infantil la más vulnerable. Existe una alta probabilidad de que estos niños estén incorrectamente vacunados y, por lo tanto, expuestos a agentes infecciosos a los que la población autóctona es inmune. En estas situaciones, debemos realizar una valoración médica inicial y una adecuada supervisión del estado vacunal, para administrar las vacunas mediante pautas aceleradas y conseguir una actualización de su carnet de vacunación de acuerdo con el calendario de inmunizaciones de nuestra comunidad autónoma.

Para elaborar este trabajo se han consultado guías de atención al inmigrante de varias comunidades autónomas de nuestro país, las recomendaciones americanas del comité de inmunizaciones y, sobre todo, se han tenido en cuenta las recomendaciones del Comité Asesor de Vacunas (CAV) de la Asociación Española de Pediatría (AEP) de 2008.

Palabras clave. Cobertura Vacunal. Migración Internacional. Niño.

\section{ABSTRACT}

Management of immigrant children: Vaccination calendar

The immigrant population in Castilla-La Mancha, Spain has multiplied in the last five years. The incorporation of immigrants has progressed very rapidly in the last few years with the greatest increase being in 2001. The immigrants often come from depressed socio-economic communities and the child population is the most vulnerable. There is a high probability that these children have not been adequately vaccinated and, therefore, are exposed to infectious agents to which the indigenous population is immune. For these children, we should perform an initial medical assessment and a review of their vaccine status in order to administer vaccines in an accelerated regimen. Thus bringing their vaccination record card up to date with the immunization calendar in our autonomous community. In order to carry out this work we have consulted guidelines on immigrant care in several autonomous communities in Spain, the American recommendations of the immunization committee and, in particular, the 2008 recommendations of the Vaccine Assessment Committee (CAV) and the Spanish Paediatric Society (AEP) have been taken into account.

Key words. Immunization Coverage. Emigration and Immigration. Child.

\section{INTRODUCCIÓN}

En los últimos años, el mundo ha asistido a un importante aumento de flujos migratorios. Nuestro país se ha transformado en un receptor importante de inmigrantes en un periodo corto de tiempo y además se ha convertido en el segundo país del mundo en adopciones internacionales ${ }^{1}$.

En cuanto a la procedencia de la inmigración en España, en un 35\% de los casos proceden de Sudamérica (Ecuador, Colombia y Perú), el 23\% de África (Marruecos), el $32 \%$ de origen europeo comunitario (Rumania), el $3 \%$ de Europa no comunitaria y el $6.5 \%$ de origen asiático (China) ${ }^{2}$. 
En muchos casos, los programas de vacunación de los países de origen son distintos al nuestro o los niños están parcialmente inmunizados, por lo que precisan valoración individualizada. Los calendarios vacunales de sus países de procedencia están basados en las vacunas mínimas recomendadas por la OMS (no incluyen vacunas frente a $\mathrm{H}$. influenzae tipo b, meningococo $\mathrm{C}$, heptavalente frente a neumococo, etc.). Proceden de comunidades socioeconómicas deprimidas donde las coberturas vacunales son bajas (sobre todo en África, donde pueden llegar a ser inferiores al 50\%). Las infraestructuras, en muchos casos, son deficientes para mantener la cadena de frío, por lo que es esperable una menor efectividad de las vacunas ${ }^{1,3,4}$.

El pediatra de atención primaria es una pieza clave en la salud de las familias inmigrantes, porque en muchas ocasiones representa el primer contacto con el sistema sanitario, sin embargo, la precaria situación legal y laboral en nuestro país hace que el contacto con los servicios de salud sea a través de urgencias y que los inmigrantes no acudan en primera instancia al centro de salud donde se llevan a cabo las inmunizaciones.

\section{CONCEPTO DE NIÑO INMIGRANTE}

Según la Asociación Americana de Pediatría, deberíamos considerar como niño inmigrante a "todo niño de otro país, con situación legalizada o no, refugiado y/o procedente de adopción internacional, así como los que se desplazan por cortos periodos de tiempo, de paso por el país o en programas vacacionales".

En España, todo niño inmigrante tiene derecho a la asistencia sanitaria según Ley Orgánica 4/2000, de 11 enero, sobre Derechos y Libertades de los Extranjeros en España ${ }^{5}$.

\section{PROTOCOLO DE ACTUACCIÓN}

Debemos conseguir, en el menor tiempo posible, que los inmigrantes reciban las mismas vacunas que los autóctonos, para que no existan bolsas de población susceptible. Hay que evitar oportunidades perdidas de vacunación, plantear estrategias a través de trabajadores sociales y hacer uso de pautas aceleradas y vacunas combinadas ${ }^{4}$.

Se administrarán primero las vacunas que inmunicen frente a patologías de mayor riesgo con relación a la edad del sujeto y epidemiología del entorno ${ }^{6}$.

\section{PRIMERA VISITA DEL NIÑO INMIGRANTE}

En primer lugar hay que realizar una anamnesis detallada (país de procedencia, inmunizaciones previas, etc.), junto con una exploración física completa que incluya peso, talla y perímetro cefálico, si es menor de 2 años. Se puede buscar cicatriz a nivel del deltoides para comprobar si ha sido vacunado, en su país de origen, de la vacuna contra la tuberculosis (BCG).

En esta primera visita podemos realizar además un mantoux, según recomendaciones de la Asociación Española de Infectología Pediátrica ${ }^{7}$.

La valoración de los niños "inmigrantes" no se puede presentar como protocolo con pautas estrictas, ya que depende del tiempo que lleva residiendo en España y del país de procedencia.

Solicitaremos pruebas de cribado básicas:

- Hemograma y reticulocitos: en busca de hemoglobinopatias.

- Bioquímica básica con estudio de hierro.

- Sedimento urinario: si aparece hematuria tendríamos que descartar esquistosomiasis.

- Parásitos intestinales: si aparece eosinofilia en el hemograma, nos orientará en la búsqueda de parásitos intestinales. Se considera que cuando un niño lleva residiendo en España más de un año las posibilidades de encontrarlos desaparecen, salvo casos sintomáticos o especiales (Strongyloides spp. y Schistosoma spp.).

- Serologías: se solicitarán serologías frente a VHB, VHC, VIH y Lues, según la zona de origen.

Se deben solicitar además pruebas específicas según sospecha clínica, como gota gruesa si consulta por fiebre, para descartar paludismo. La posibilidad de transmisión a la población local de estas enfermedades exóticas es muy improbable, tanto por el bajo número de casos, como por la inexistencia de vectores y/o hospedadores intermediarios adecuados ${ }^{9}$.

Se valorará documentación de vacunas administradas en el país de origen o en otros lugares de nuestro país donde haya residido previamente y se actualizará su calendario vacunal de forma individualizada ajustándolo al de nuestra comunidad autónoma.

\section{NIÑOS PARCIALMENTE INMUNIZADOS}

En la supervisión del calendario de vacunación, podemos encontrar datos que hacen pensar en registros falsificados, como fechas de vacunación anteriores a la fecha de nacimiento del niño ${ }^{10}$. Si acreditamos 
su fiabilidad, las vacunas serán consideradas como válidas independientemente del tiempo transcurrido desde la última dosis, de manera que procederemos a completar las dosis necesarias teniendo en cuenta la edad del niño (tabla 1).

Situaciones que encontramos frecuentemente en las cartillas de vacunación de los inmigrantes:

- La vacuna contra el sarampión administrada en estos países es, a menudo, monovalente, por lo que no aporta protección frente a Rubéola y Parotiditis ${ }^{11}$.

- La vacunación frente a Hepatitis B en hijos de madres portadoras se aplica pasada la primera semana de vida, por lo que no asegura prevención de la trans- misión vertical de virus ${ }^{1,2,3}$

\section{NIÑOS NO INMUNIZADOS O DESCONOCIMIENTO DE LA SITUACIÓN}

Si no existe constancia por escrito, se considerará "no vacunado", ya que no supone ningún riesgo el vacunar a niños ya inmunizados ${ }^{3}$.

Algunas dudas se pueden resolver mediante pruebas analíticas como concentraciones de anticuerpos anti$\mathrm{HBs}$, que cuando resultan mayores de $10 \mathrm{mUl} / \mathrm{ml} \mathrm{se}$ consideran protectores frente a Hepatitis B. Por otra parte, una reacción de inflamación local importante al

\begin{tabular}{|c|c|c|c|c|c|c|c|}
\hline Edad & $1^{2}$ Visita & Almes* & A los 2 meses & A los 4 meses & A los 6 meses & $\begin{array}{c}\text { A los } 12 \\
\text { meses }\end{array}$ & $\begin{array}{c}\text { A los } 24 \\
\text { meses }\end{array}$ \\
\hline $\begin{array}{l}0-14 \\
\text { meses }\end{array}$ & $\begin{array}{l}\text { - Pentavalente } \\
\text { - Hepatitis } \mathrm{B}^{3} \\
\text { - Meningitis } \mathrm{C}^{2} \\
\text { - Rotavinus }\end{array}$ & - Hepatitis $\mathrm{B}^{\prime}$ & $\begin{array}{l}\text { - Pentavalente }{ }^{3} \\
\text { - Meningitis } \mathrm{C}^{2} \\
\text { - Neumococo }\end{array}$ & $\begin{array}{l}\text { - Pentavalente }{ }^{3} \\
\text { - Neumococo }\end{array}$ & - Hepatitis $\mathrm{B}^{\prime}$ & - Neumococo & \\
\hline $\begin{array}{l}15 \text { meses } \\
\text { a } 2 \text { anios }\end{array}$ & $\begin{array}{l}\text { - Pentavalente } \\
\text { - Hepatitis } \mathrm{B}^{3} \\
\text { - Meningitis } \mathrm{C}^{2} \\
\text { - Neumococo } \\
\text { - T. } \mathrm{V}^{+}\end{array}$ & - Hepatitis $\mathrm{B}^{\prime}$ & $\begin{array}{l}\text { - DTPa } \\
\text { - VPI } \\
\text { - Neumococos } \\
\text { - Varicela }{ }^{\circ}\end{array}$ & $\begin{array}{l}\text { - DTPa } \\
\text { - VPI }\end{array}$ & - Hepatitis $\mathrm{B}^{\prime}$ & $\begin{array}{l}\text { - VPI } \\
\text { - DTPa }\end{array}$ & $\begin{array}{l}\text { Seguir } \\
\text { calendario } \\
\text { oficial de } \\
\text { CCAA }\end{array}$ \\
\hline $\begin{array}{l}2 \mathrm{a} 6 \\
\text { anios }\end{array}$ & $\begin{array}{l}\text { - T. } \mathrm{V}^{+} \\
\text {- Pentavalente } \\
\text { - Hepatitis } \mathrm{B}^{\mathrm{s}} \\
\text { - Meningitis } \mathrm{C}^{2}\end{array}$ & $\begin{array}{l}\text { - T. } V^{*} \\
\text { - Hepatitis } \mathrm{B}^{\prime} \\
\text { - Neumococo }\end{array}$ & $\begin{array}{l}\text { - VPI } \\
\text { - DTPa } \\
\text { - Varicela }\end{array}$ & $\begin{array}{l}\text { - DTPa } \\
\text { - VPI }\end{array}$ & - Hepatitis $\mathrm{B}^{\prime}$ & $\begin{array}{l}\text { - VPI } \\
\text { - DTPa }\end{array}$ & \\
\hline $\begin{array}{l}7 \text { a } 18 \\
\text { ainos }\end{array}$ & $\begin{array}{l}-T \cdot V^{+} \\
\text {- Td } \\
\text { - VPI } \\
\text { - Hepatitis } \mathrm{B}^{\prime} \\
\text { - Meningitis } \mathrm{C}^{2}\end{array}$ & $\begin{array}{l}\text { - T.V } \\
\text { - Td } \\
\text { - VPI } \\
\text { - Hepatitis } \text { B' }^{\prime} \\
\text { - Varicela }\end{array}$ & & & $\begin{array}{l}\text { - VPI } \\
\text { - Hepatitis B' }\end{array}$ & $-T d$ & \\
\hline $\begin{array}{l}>18 \\
\text { anios }\end{array}$ & $\begin{array}{l}\text { - T. } V^{+} \\
\text {- Td } \\
\text { - VPI } \\
\text { - Hepatitis } \text { B' }^{\prime}\end{array}$ & $\begin{array}{l}\text { - Td } \\
\text { - VPI } \\
\text { - Hepatitis } \mathrm{B}^{\prime} \\
\text { - Varicela }\end{array}$ & & - Varicela ${ }^{6}$ & - Hepatitis $\mathrm{B}^{\prime}$ & $\begin{array}{l}-T d \\
\text {-VPI }\end{array}$ & \\
\hline
\end{tabular}

Tabla 1. Calendario a seguir en no vacunados. ${ }^{\star}$ Meses contados a partir de la $1^{\text {a }}$ visita. En cursiva (Neumococo, Rotavirus y Varicela) aparecen las vacunas no incluidas en el calendario oficial de Castilla La Mancha, pero que son recomendadas por la AEP.

${ }^{1}$ El esquema ideal de administración de la Hepatitis B es 0-1-6 meses, aunque se ha comprobado la eficacia de prácticamente cualquier secuencia. Ante posible contacto inminente, una pauta acelerada sería 0-1-2-12 meses.

${ }^{2}$ El número de dosis de Meningitis $C$ varía según edad de comienzo: $<12$ meses 2 dosis con una $3^{\mathrm{a}}$ dosis al $2^{\circ}$ año de vida, $\geq 12$ meses 1 dosis.

${ }^{3}$ Vacuna Pentavalente: Incluye DTPa (difteria, tétanos y tos ferina acelular), Hib (Haemophilus influenzae tipo B) y VPI (vacuna de la polio inactivada). Las dosis de Haemophilus Influenzae varía según la edad de comienzo: <7 meses 4 dosis, 7-11 meses 3 dosis, $12-14$ meses 2 dosis, $\geq 15$ meses 1 dosis. No obstante, los niños pertenecientes a grupos de riesgo recibirán dos dosis con un intervalo de 8 semanas.

${ }^{4}$ En la vacuna triple vírica (T.V.) no se contabilizan las dosis administradas antes de los 12 meses.

${ }^{5}$ Neumococo: el número de dosis varía según la edad de comienzo: <7 meses 4 dosis, 7-11 meses 3 dosis, 11-23 meses 2 dosis, 24-60 meses 1 dosis. No indicada en $>60$ meses.

${ }^{6}$ La vacuna de la Varicela se puede administrar a partir de los 12 meses. Incluida en el calendario de Castilla La Mancha a los 11 años en caso de no haber padecido la enfermedad.

${ }^{7}$ La vacuna frente a Rotavirus se puede administrar entre las 6 semanas y los 6 meses de vida, con 2 ó 3 dosis (según marca comercial). 


\begin{tabular}{l|cccccc}
\hline Edad & T.Vírica & DTPa & H.Influenzae B & Polio & Hepatitis B & Meningitis C \\
\hline$<24$ meses & 1 & 4 & $1-4^{* *}$ & 4 & 3 & $1-2^{\star \star *}$ \\
24 meses-6 años & 2 & $4-5$ & 1 & 4 & 3 & 1 \\
7-18 años & 2 & $3(T d)^{\star}$ & $3(T d)^{\star}$ & 3 & 3 & 1 \\
$>18$ años & 1 & 3 & 3 & 3 \\
\hline
\end{tabular}

Tabla 2. Número de dosis recomendadas para considerar correcta la vacunación. * A partir de los 7 años sólo se administran los componentes Tétanos y Difteria. ${ }^{* *}$ Las dosis administradas frente a H. Influenzae tipo B varían según la edad de comienzo de la vacunación: $<7$ meses 4 dosis, 7-11 meses 3 dosis, 12-14 meses 2 dosis, $>15$ meses 1 dosis.

*** Las dosis administradas frente a Meningitis $C$ varían según la edad de comienzo de la vacunación: $<12$ meses 2 dosis con un intervalo de 4 semanas y una $3^{a}$ dosis al $2^{\circ}$ año de vida, $>12$ meses 1 dosis.

\begin{tabular}{l|l}
\hline Tipos de antígenos & Intervalo mínimo \\
\hline Dos o más Ag. inactivados & Simultáneamente o con cualquier intervalo \\
Ag. inactivado y Ag. atenuado & Simultáneamente o con cualquier intervalo (1) \\
Dos o más Ag. atenuados & Simultáneamente o separados al menos 4 semanas(2) \\
\hline
\end{tabular}

Tabla 3. Intervalos entre dosis de distintas vacunas. Ag. (antígeno), VPO (vacuna de la polio oral). (1) La única excepción es la administración de vacuna Anticolérica (inactivada) y vacuna contra la Fiebre Amarilla (atenuada) que han de separarse al menos tres semanas. (2) La VPO puede administrarse antes, simultáneamente o después de la vacuna triple vírica, varicela y vacuna oral contra la Fiebre Tifoidea. * De las vacunas recomendadas de manera sistemática sólo son antígenos atenuados, es decir vacunas vivas, la triple vírica y la vacuna contra la varicela, siendo todas las demás inactivadas.

administrar la vacuna de tétanos-difteria significa que ya ha recibido dosis previas.

\section{INMUNIZACIONES SISTEMATICAS (tablas 1 y 2)}

Se administrará el máximo número de vacunas en la primera visita (tabla1) y se completarán las dosis necesarias (tabla 2) teniendo en cuenta los intervalos necesarios entre vacunas:

- Dosis de de una misma vacuna: el intervalo mínimo entre dos dosis es de un mes, no existiendo intervalo máximo.

- Dosis de distintas vacunas (tabla 3): se pueden administrar varias vacunas, siempre que se realice en puntos anatómicos diferentes. Si fuera necesario aplicarlas en el mismo miembro, deben estar suficientemente separadas, al menos $3-5 \mathrm{~cm}$.

\section{VACUNA TRIPLE VÍRICA}

Se administra aunque existan antecedentes de cualquiera de las enfermedades que previene. El Sarampión es endémico en países en vías de desarrollo. En estos países, se administra sarampión monovalente y de forma precoz (antes del año de edad), de manera que el antígeno vacunal es neutralizado por anticuerpos maternos, por lo que, en estos niños, no se contabilizan las dosis recibidas antes de los 12 meses, ni la vacuna monovalente frente a sarampión $n^{1,3}$. Por lo tanto, habrá que administrar la vacuna triple vírica a los 15 meses. Entre los 4 y 19 años se considera vacunación adecuada a los que han recibido 2 dosis separadas un mínimo de 28 días.

Existe una alta tasa de mujeres inmigrantes susceptibles a rubéola en edad fértil (especialmente sudamericanas). Es relativamente fácil la formación de bolsas de población susceptible con la posibilidad de aparición de brotes, como el de la Comunidad de Madrid durante el año 2005 (496 casos desde 3-10-04 hasta 31-8-05, y lo que es más grave, 5 casos de rubéola congénita).

\section{VACUNA CONJUGADA CONTRA MENINGOCOCO C}

No existe esta vacuna en la mayoría de países en vías de desarrollo y se debe administrar en inmigrantes menores de 20 años. En menores de un año administrar 2 dosis con un intervalo de 2 meses y refuerzo posterior entre los 12 y 24 meses. Si es mayor de un año recibirá una sola dosis.

\section{VACUNA DIFTERIA-TÉTANOS-TOS FERINA (DTP)}

Según el CAV se deben administrar vacunas combinadas acelulares (DTPa) con menos reacciones secundarias que las de células enteras (DTPe).

Se puede revacunar a pesar de dosis previas, pero suele haber reacción local a partir de la $4^{\circ}$ dosis. Se puede plantear administrar un total de 4 dosis, puesto 
que es probable que hayan recibido alguna dosis en su país de origen. Pauta: 3 dosis separadas por 2 meses, $4^{\text {a }}$ dosis a los 12 meses de la tercera. A partir de los 7 años se utilizará el preparado sin componente pertussis (Td) según pauta de adultos (0-1-6 meses) en la primovacunación.

La comercialización de vacunas acelulares con componente antigénico reducido de $B$. pertussis $(\mathrm{pa})$, en forma de vacuna combinada (dTpa), hace recomendable su utilización como dosis de recuerdo en los adolescentes y los adultos ${ }^{12}$.

\section{VACUNA DE LA VARICELA}

Los niños inmigrantes suelen llegar a nuestro país sin haber pasado esta enfermedad, ya que en sus países de origen se padece en edades más tardías que en nuestro medio. Es una vacuna de virus vivos atenuados. Según el CAV, habría que realizar vacunación universal entre los 12 -15 meses o vacunación selectiva a los 11-12 años si no han padecido la enfermedad previamente. Nuevas recomendaciones del CAV, en 2008 , recomiendan administrar una $2^{\mathrm{a}}$ dosis de vacuna a los 3-6 años simultáneamente con la $2^{a}$ dosis de triple vírica ${ }^{13}$.

\section{VACUNA ANTINEUMOCÓCICA CONJUGADA}

Según recomendaciones del CAV de la AEP, la vacuna antineumocócica conjugada heptavalente se debe administrar en menores de 2 años $^{13}$.

El número de dosis a recibir está en función de la edad: 3 dosis en menores de 7 meses con refuerzo al $2^{\circ}$ año de vida y 2 dosis entre 7-11 meses de vida con refuerzo posterior en el $2^{\circ}$ año. Entre los 12 y 24 meses se administran 2 dosis y a partir de los 2 años 1 dosis.

\section{VACUNA ANTIPOLIOMIELÍTICA}

La OMS emitió el certificado de erradicación de la polio en Europa en junio de 2001, por lo que se considera a esta región libre de polio salvaje. Esto ha motivado la sustitución de la vacuna oral de virus vivos (VPO) por la vacuna inactivada intramuscular (VPI), introducida en enero de 2004 en España. Si han recibido polio oral en su país, la vacunación de los niños inmigrantes se actualizará con la vacuna de polio inactivada, hasta recibir las dosis necesarias según la edad (tabla 1 y 2).

En caso de declaración de brote, la población de riesgo se vacunaría con VPO, dada su ventaja de vacunación colectiva a través de su eliminación por heces ${ }^{14}$.

\section{VACUNA HAEMOPHILUS INFLUENZAE TIPO B}

Deben vacunarse los niños menores de 5 años ${ }^{13}$. Esta vacuna ha sido incluida por la OMS desde Marzo de 2002. Actualmente está introducida en Latinoamérica (salvo Ecuador). No se administra en África ni Asia.

Pauta: en menores de 6 meses, 3 dosis con un intervalo de 2 meses (si pauta acelerada, 4 semanas) y refuerzo posterior a los 12-18 meses. Entre 6 y 12 meses, 2 dosis con un intervalo de 2 meses y refuerzo posterior a los 12-18 meses. Los mayores de 12 meses recibirán una sola dosis.

\section{VACUNA ANTI HEPATITIS B}

Introducida en más de 140 países, sin embargo se encuentran bajas coberturas vacunales. En África subsahariana se mantienen tasas de incidencia de portadores de hepatitis $\mathrm{B}$ en población general por encima del $8 \%$. Es aconsejable realizar serología prevacunación, para descartar portadores crónicos de VHB, a niños provenientes de países de alta endemicidad (África, Asia) y/o pertenecer a grupos de riesgo.

\section{VACUNA ANTI HEPATITIS A (HA)}

Indicada en inmigrantes que pertenezcan a grupos de bajo nivel social que viajen a país de origen con alta prevalencia de hepatitis $A^{3,4}$.

Pauta: dos dosis separadas 6-12 meses. En algunas comunidades españolas se utiliza antihepatitis $A+B$ en pauta de tres dosis ( 0,1 y 6 meses). Esta vacuna no se recomienda en menores de 1 año.

\section{ESTRATEGIAS PARA MEJORAR LA EFECTIVIDAD DE UN PROGRAMA DE VACUNACIÓN EN NIÑOS INMIGRANTES}

Para conseguir mejorar las coberturas vacunales en la población infantil inmigrante, y ante la existencia de población susceptibles a enfermedades que se pueden prevenir, se recomiendan estrategias como:

1. Disponibilidad de centro de salud.

2. Aprovechar cualquier oportunidad de verificar y poner al día las vacunas.

3. Utilizar pautas aceleradas de vacunación.

4. Mensajes recordatorios y cita automática si faltan a la vacunación.

5. Coordinación con otros niveles, colegios, guarderías, servicios sociales, colonias urbanas etc.

6. Desestimar falsas contraindicaciones de la vacunación, como estar recibiendo tratamiento antibióti$\mathrm{CO}^{15}$, etc. 
7. En caso de duda, es mejor administrar dosis de más que no completar las inmunizaciones.

8. Exigir documentación sobre inmunizaciones recibidas.

9. Investigar para detectar portadores de enfermedades transmisibles y tomar las medidas profilácticas oportunas

\section{PÁGINAS WEB DE INTERÉS DEL VIAJERO}

1. Centers for Disease Control and Prevention (www. cdc.gov/travel).

2. World Health Organization (www.who.int/ith).

3. Ministerio de Sanidad y Consumo (www.msc.es).

4. Asociación Española de Pediatria (www.aeped.es) (www.vacunasaep.org).

5. Internatinal Society of Travel Medicine (www.istm. org).

6. European Center for Disease Prevention and Control (www. ecdc.eu.int).

7. Program for Monitoring Emerging Diseases (www. promedmail.org)

\section{BIBLIOGRAFÍA}

1. Santos Sebastián MM, Gonzalez J, Gómez Campderá JA. Vacunación en niños inmigrantes. Acta Pediatr Esp 2008; 66(7):337-340.

2. Hidalgo Vicario I, Gancedo M, Güemes Hidalgo M. Cobertura vacunal. Manual de Atención al Paciente inmigrante. Madrid: Comunicación Médica; 2007. p. 109-131.

3. Comité de Enfermedades Infecciosas de American Academy of Pediatrics. "Red Book" de Enfermedades Infecciosas en Pediatría. 26ª ed. P. 36-7, 76-81,104-5, 203-5, 314.
4. Farjas Abadía MP, Zubizarreta Alberdi R. Manual de vacunación. Vacunación en el inmigrante; 2008. Guía clínica Fisterra. Disponible en: http://www.fisterra.com/vacunas/ inmigrante.asp.

5. Cortés Rico O, Aparicio Hernán A, Montón Álvarez JL. Valoración inicial del niño inmigrante. Pediatría Integral 2005; IX:725-33.

6. Picazzo JJ (ed). Guía práctica de vacunaciones. Madrid: Marco Gráfico S.L.; 2002. Disponible en: http:/www.vacunas.net.

7. Grupo de Trabajo de Tuberculosis de la Sociedad Española de Infectología Pediátrica. Interpretación de la prueba de tuberculina en niños. An Pediatr (Barc) 2003; 59(6):582-5.

8. Montesdeoca A, Poch J, Mateos M, Mesa Y, Sancho A, Herranz M.T. Valoración del niño inmigrante. Monografías Emergencias 2008 (En prensa).

9. Hernández Cabrera M, Carballo Rastrilla S, Carranza Rodríguez C, Pérez Arellano JL. Infecciones en el niño inmigrante en España. Escenarios de actuación. Enf. Emerg 2003; 5(3):147-59.

10. García Sánchez N, de Juan Martín F. La vacunación del niño inmigrante y del procedente de adopción internacional. Un gran reto. Boletín SPARS 2004; 34(2):46-54.

11. Lee PJ. Vaccines for travel and international adoption. Pediatr Infect Dis J 2008; 27(4):351-4.

12. Campins M, Moraga F. Vacunación frente a la tosferina en el adolescente y el adulto. Enferm Infecc Microbiol Clin 2008; 26:12-9.

13. Bernaola Iturbe E, Giménez Sánchez F, Baca Cots $M$, de Juan Martín F, Diez Domingo J, Garcés Sánchez M et al. Calendario vacunal de la Asociación Española de Pediatría: recomendaciones 2008. An Pediatr (Barc) 2008; 68(1):639.

14. Arístegui Fernandez J, Gonzalo de Liria CR. Protocolos AEP. Infectología. Calendarios Vacunales; 2000. p. 37-44.

15. General Recomendations on Inmunization: Recommendation of the Advisory Committee on Immunization Practices (ACIP). MMWR 2006; 55 (RR-15):1-48. 